\title{
Corela
}

Cognition, représentation, langage

HS-22 | 2017

Prédication et prépositions en anglais

\section{La mise en relation par la prédication et les prépositions en anglais : complémentarité, convergences et différences dans quelques approches théoriques}

\section{Laurence Vincent-Durroux}

\section{OpenEdition}

Journals

Édition électronique

URL : http://journals.openedition.org/corela/4988

DOI : $10.4000 /$ corela.4988

ISSN : 1638-573X

Éditeur

Cercle linguistique du Centre et de l'Ouest - CerLICO

Référence électronique

Laurence Vincent-Durroux, «La mise en relation par la prédication et les prépositions en anglais complémentarité, convergences et différences dans quelques approches théoriques », Corela [En ligne], HS-22 | 2017, mis en ligne le 15 février 2018, consulté le 19 avril 2019. URL : http:// journals.openedition.org/corela/4988 ; DOI : 10.4000/corela.4988

Ce document a été généré automatiquement le 19 avril 2019

\section{(†) (2)}

Corela - cognition, représentation, langage est mis à disposition selon les termes de la licence Creative Commons Attribution - Pas d'Utilisation Commerciale - Partage dans les Mêmes Conditions 4.0 International. 


\title{
La mise en relation par la prédication et les prépositions en anglais : complémentarité, convergences et différences dans quelques approches théoriques
}

\author{
Laurence Vincent-Durroux
}

1 Dès l'Antiquité, les philosophes grecs se sont préoccupés de caractériser le langage, et, en rapport avec la pensée et son fonctionnement par mises en relation, ils envisageaient déjà le langage comme résultant d'assemblages. Ainsi, Platon, dans Le Sophiste, décrit ainsi le discours :

Celui-ci, en liant les verbes et les noms, donne ainsi des indications sur les choses, aussi bien sur celles qui se produisent, ou sur celles qui ont été produites ou qui se produiront, et non seulement il leur applique une détermination mais il achève quelque chose. C'est pour cela que non seulement il nomme, mais aussi qu'il « lie ", et c'est à cet entrelacement que nous appliquons le nom de discours.

2 Le langage est désormais fondamentalement conçu comme une activité cognitive de mise en relation : sont ainsi mis en relation des concepts et des référents, des signifiés et des signifiants, mais aussi des notions entre elles par l'activité de prédication au sens le plus large.

Dans la langue, cette activité se manifeste par des éléments et des formes de natures diverses, telles que la conjugaison (qui explicite de manière typique le lien entre le sujet et le verbe), l'accord possible entre adjectifs et noms, ou encore des mots qui indiquent que des relations ont été nouées : conjonctions, prépositions...

4 Ce recueil porte sur l'activité linguistique de mise en relation et sur les traces qu'elle laisse dans la langue, en circonscrivant le propos à la prédication et aux prépositions. Il 
ne s'agit pas tant de juxtaposer deux thématiques relevant, a priori, pour l'une du domaine verbal, pour l'autre du domaine nominal, en vue d'assurer une perspective sur la langue qui se voudrait large, que de prendre en compte des phénomènes de langage auxquels la mise en relation procure une complétude: ainsi, le prédicat est un dit « incomplet » (Diogène Laërce, cité par J.-M. Merle, ce volume), en attente de prédication par la mise en relation avec un sujet ; la préposition quant à elle est « traditionnellement présentée comme un terme subordonnant» (Riegel, 1998) et donc dépendant. La préposition met en relation ce qu'elle introduit et ce qui la précède, et peut être décrite comme un "prédicat à deux places" (Amiot [2006] 2012). Sa fonction est fondamentalement prédicative (Franckel \& Paillard, $2007: 18$ ) et la préposition peut être caractérisée de «relateur » (Hagège, 1997 et Huddleston \& Pullum, 2002, cités par J.-M. Merle, ce volume), motivant le rapprochement effectué dans ce recueil.

Comment différentes théories linguistiques contemporaines abordent-elles les phénomènes de mise en relation? Comment, ensemble, peuvent-elles mieux les caractériser et les expliquer? Ce recueil a pour objet la mise en regard des réponses de différentes approches théoriques à la question de la mise en relation. La langue étudiée ici est plus particulièrement la langue anglaise.

Pour tenter de répondre à ces questions, deux journées d'étude soutenues se sont déroulées à l'Université Paul-Valéry, Montpellier 3 (Equipe EMMA, Etudes Montpelliéraines du Monde Anglophone). Une journée a été consacrée à la prédication, une autre aux prépositions. Les textes présentés ici émanent de ces journées, ce qui a permis à leurs auteurs d'intégrer parfois des remarques soulignant des points communs, des différences ou des points de vue complémentaires apparus lors des présentations orales, discutés lors des tables rondes et développés ci-dessous (paragraphe 3).

7 Le regroupement effectué ici ne prétend pas offrir une image exhaustive des approches actuelles, d'autant plus que quelques invités ont été empêchés. En revanche, il expose différentes manières de traiter de la mise en relation, met ses enjeux en évidence et fait apparaître la richesse qui découle d'un regard à la fois analytique et synthétique permis par le rapprochement de cadres théoriques différents.

8 Les théories représentées ici ont en effet des points d'entrée dans le langage qui les distinguent : l'observable qu'est la syntaxe, pour la grammaire générative ; l'énonciateur et les choix qu'il effectue, choix étudiés dans le cadre de la théorie des opérations prédicatives et énonciatives; l'activité cognitive pour la linguistique cognitive et la grammaire des constructions.

Chaque auteur rend accessible au lecteur les concepts de la théorie utilisée et illustre son propos par l'analyse d'un même corpus d'étude, extrait d'un roman d'Ann Tyler pour la prédication et d'un roman de Margaret Atwood pour les prépositions. Ces extraits sont reproduits avant chaque partie.

\section{Quelques approches de la prédication}

10 La première partie est consacrée à la prédication, qui serait associée à une opération cognitive profonde. Des travaux de neurologie cités par Gilles Col (2008) montrent que la structure prédicat-argument constitue le centre de représentations mentales prélinguistiques. Mais qu'est-ce que le prédicat? Et qu'est-ce que la prédication? 
11 Le concept et son interprétation ont évolué depuis l'Antiquité, ce dont rend compte tout particulièrement la première partie de la contribution de Jean-Marie Merle, qui présente trois appréhensions possibles de la prédication et montre que les approches actuelles sont sous-tendues par l'interprétation de Platon et des Stoïciens, puis par celle d'Aristote. Le lecteur pourra utilement se reporter aux références citées, renvoyant à des volumes spécialisés sur l'histoire du concept de prédication, notamment Merle (dir., 2008).

12 La contribution de Jean-Marie Merle donne ensuite le point de vue de la Théorie des opérations prédicatives et énonciatives (TOPE). Dans ce cadre, le prédicat occupe une place centrale par rapport à laquelle le sujet est incident, selon une perspective néoplatonicienne. Jean-Marie Merle développe notamment les rapports étroits entre prédication et (pluri)-modalité, prédication, bi-prédication et co-prédication, subordination, relativisation et enchâssement, pour parvenir à une définition large de la prédication comme "structuration modalisée qui fonde l'énoncé ».

Jean-Charles Khalifa présente l'analyse de la prédication en grammaire générative. La prédication y est habituellement associée à la catégorie du verbe. Elle intervient au moment où l'argument externe au verbe devient sujet et où se mettent en place les coordonnées temporelles, complétant ainsi le prédicat. En cas de sujet non exprimé, TO est alors une trace sensible de l'expression temporelle, analyse qui rejoint celle de la théorie méta-opérationnelle pour laquelle TO est fondamentalement prédicatif. Des verbes tels que SEEM rendent problématique l'analyse de la prédication et nécessitent le recours au mouvement de montée du sujet syntaxique, analyse que Jean-Charles Khalifa propose d'utiliser pour rendre compte de la prédication dans les énoncés avec $B E$ copule.

Jean-Rémi Lapaire montre que la linguistique cognitive voit la prédication de manière constructionnelle, par référence à la "grammaire des constructions" (Construction Grammar). Dans ce cadre, il revient au verbe de "scénariser les événements et de signaler l'angle sous lequel ceux-ci sont perçus et conçus ». La combinatoire verbale, à laquelle la prédication pourrait se rattacher, implique tout autant la grammaire que le lexique; ensemble, ils constituent un répertoire pré-existant de "patrons structuraux » et de "schèmes sémantiques" dans lequel puise l'énonciateur. Ce répertoire de structures symboliques offre au sujet cognitif le moyen d'organiser ses représentations d'une scène. Après avoir mis en évidence l'apport de B.L. Whorf à la grammaire des constructions, Jean-Rémi Lapaire montre que la conception de la prédication s'est développée «en direction de la structure argumentale que chaque verbe dessine et que les constructions achèvent de configurer voire de reconfigurer $"$.

\section{Quelques approches des prépositions}

La seconde partie est consacrée aux prépositions. Les prépositions constituent une catégorie de mots grammaticaux et sont assorties d'une valeur lexicale; la valeur grammaticale qu'elles expriment est la mise en relation entre des termes. Les contributions présentées ici portent sur des prépositions réalisées phonologiquement et graphiquement; elles ne se sont pas penchées sur le statut particulier de la préposition non réalisée phonologiquement, notamment en anglais où la préposition $\varnothing$ peut être mise en système avec of.

Dans les diverses langues, les prépositions sont en nombre réduit mais les effets de sens de chaque préposition sont souvent nombreux. Si les apprenants de langue étrangère 
sont parfois confrontés à des listes d'emplois pour chaque préposition, les linguistes cherchent à unifier ces emplois autour d'une valeur sémantique commune, centrale et fondamentale, dont dériveraient tous les emplois d'une même préposition. Cette valeur pourrait trouver son origine dans un ancrage spatial ou spatio-temporel selon les hypothèses qui s'appuient sur une réalité cognitive sous-jacente au langage (François et al., dir., 2009) et qui ancrent les concepts linguistiques dans l'expérience et les perceptions de l'être humain.

17 Par une mise en perspective diachronique des prépositions de l'anglais, la contribution d'Anne Mathieu étayent les hypothèses des théories linguistiques cherchant à établir une valeur centrale pour chaque préposition. Anne Mathieu développe en effet une approche sémantique des prépositions présentes dans le corpus d'étude, pour lesquels les emplois spatiaux, temporels et abstraits sont attestés dès le Vieil-Anglais. Anne Mathieu montre que les procédés qui rendent compte des évolutions constatées dans les usages d'une même préposition sont d'une part l'influence d'autres langues, en particulier le français, d'autre part l'extension d'emplois préalables. Ainsi, by a été progressivement employé devant les compléments d'agent par extension de by à valeur instrumentale; l'emploi de of pour relier un objet à l'une de ses caractéristiques se fit par grammaticalisation; enfin l'emploi de for devant une durée relève de l'extension d'emplois abstraits. Cet apport diachronique met en lumière et développe les procédés sémantiques sur lesquels certaines approches théoriques se fondent, notamment la linguistique cognitive. La contribution de Jean-Rémi Lapaire s'inscrit dans une résonnance particulière avec celle d'Anne Mathieu, valorisant le fait que les connaissances en diachronie soutiennent les hypothèses théoriques.

Jean-Rémi Lapaire apporte le point de vue de la grammaire cognitive. Sachant qu'un état antérieur de la linguistique cognitive se dénommait space grammar, cette approche linguistique est particulièrement propice à l'analyse des prépositions, dont l'origine est souvent la spatialité. Les principes fondateurs de la grammaire cognitive sont d'abord décrits, en rapport avec le besoin humain de représenter l'expérience spatio-temporelle. Le corps humain et la représentation que le locuteur s'en fait sont centraux mais il faut aussi intégrer les caractéristiques culturelles dans lesquels s'ancrent les usages linguistiques. Dans ce cadre théorique, l'analyse des prépositions tient compte de leur valeur, avec une réponse convaincante à la question de l'invariant, mais aussi de la construction à laquelle elles se rattachent.

Jean-Marie Merle caractérise les prépositions selon une approche énonciative, notamment sous l'angle des repérages. Une préposition est en effet caractérisée par sa fonction de relateur qui permet de relier une facette d'un terme à un autre. J.-M. Merle présente les différentes facettes pouvant être saisies, d'un référent, d'un procès, d'un événement ou encore d'un état de fait. Dans son étude, J.-M. Merle propose d'étendre la catégorie des prépositions aux « conjonctions » qui introduisent une proposition de type nominal, ainsi qu'aux particules adverbiales pouvant être glosées par un groupe prépositionnel. Cette extension s'accompagne d'une réflexion sur la possibilité d'assimiler la préposition à un prédicat doté d'une valence variable. Les prépositions sont également traitées en termes de repérages, qui peuvent être de natures structurelle, énonciative ou encore sémantico-référentielle.

20 Jean-Charles Khalifa souligne qu'en première analyse, la grammaire générative envisage les prépositions comme une des quatre grandes catégories lexicales, caractérisée par l'absence de traits verbaux ou lexicaux, la présence de Spécifieurs restreints aux adverbes 
de degrés et d'intensité, et de compléments sous forme de groupes nominaux. Une seconde analyse effectue des distinctions supplémentaires entre les prépositions fonctionnelles, les prépositions semi-lexicales, les adverbes et les particules, dans le cadre plus large d'un domaine prépositionnel qui offre une analyse unifiée de quatre souscatégories, discriminées notamment par la présence ou l'absence de Spécifieur dans leur structure.

\section{Pour une lecture synthétique}

\section{Mise en relation par la prédication}

Muni de ces différentes analyses, le lecteur peut aborder le concept de prédication en mettant en rapport ses enjeux et les approches théoriques. Les différentes théories s'accordent sur le rôle fondateur de la prédication tout en définissant celle-ci de façon différente.

Selon l'étude menée dans le cadre de la TOPE, il s'agit d'une «structuration modalisée qui fonde l'énoncé ", dans laquelle le relateur est central. Il y a prédication lorsqu'il y a instanciation des places d'arguments de ce relateur. J.-M. Merle pose alors la question du rôle du prédicat par rapport au sujet, lors de l'opération de prédication : le sujet est-il un apport ou un support? Les arguments en faveur de l'antériorité conceptuelle et référentielle du sujet sont convaincants et conduisent à favoriser l'interprétation du sujet comme support de la prédication.

L'association de la modalisation à la structuration s'explique par l'ancrage, fondamental dans cette théorie, de l'énoncé dans une situation d'énonciation. Cet ancrage est assorti d'opérations énonciatives, dont la modalisation fait partie. De plus, la prise en compte de la modalisation dans la définition même de la prédication permet d'analyser les énoncés sans relation prédicative. Si opérations prédicatives et opérations énonciatives sont présentées de manière distincte dans la théorie, l'analyse de J.-M. Merle rappelle qu'elles n'en sont pas moins combinées pour produire un énoncé.

Pour la grammaire générative, il y a prédication lorsque l'argument externe devient sujet et que l'événement est muni de ses coordonnées temporelles. Il est à noter que les coordonnées temporelles sont incluses dans la modalisation revendiquée par la TOPE et que les deux théories se rejoignent de ce point de vue.

Enfin, en grammaire cognitive, la prédication est à comprendre comme une construction, convoquée parmi des structures symboliques à la disposition du locuteur et organisatrices de représentations inscrites dans un déroulement temporel. Une capacité cognitive de l'être humain est en effet de pouvoir inscrire les événements dans le temps.

Pour toutes les approches présentées, la prédication est un processus. Ce qui varie semble être le déroulement de ce processus et son inscription dans la temporalité. Si les coordonnées temporelles de la situation dans laquelle s'envisage l'événement participent toujours à la prédication, il semble y avoir des conceptions divergentes quant à la réalisation du processus même de prédication, selon que l'on se place dans la perspective de constructions préétablies (les «patrons» de la linguistique cognitive abordée sous l'angle de la grammaire des constructions), qui ne sont pas « composées dans l'instant de parole » (J.-R. Lapaire), qui ne sont donc pas à construire mais à récupérer, ou que l'on se 
place dans la perspective des autres approches, pour lesquelles il semble y avoir mise en œuvre du processus à chaque prédication.

D'une approche à l'autre, le rôle du sujet varie et cela semble dépendre du point d'entrée dans la langue, selon qu'il favorise ou non la prise en compte de la sémantique. Le sujet entre dans une « contrainte » syntaxique qui en fait mécaniquement l'argument du verbe en grammaire générative, alors que pour la TOPE le sujet est un support avec une dimension référentielle.

Etant donné le rôle contraignant attribué au sujet dans la définition même de la prédication, les auteurs se penchent sur les cas d'absence de sujet. Puisque le sujet est impliqué dans les diverses définitions de la prédication, comment la prédication peut-elle être assurée dans les propositions subordonnées infinitives, les extrapositions du sujet, les propositions coordonnées sans sujet répété ? Il s'agit alors de répondre à la question : tout énoncé émane-t-il d'une prédication ? Là encore, c'est le degré de prise en compte de la sémantique qui explique les différences entre les études. Pour la grammaire générative, qui intègre assez peu de sémantique dans l'analyse, il y a prédication dans tout énoncé. $\mathrm{Si}$ le sujet n'est pas réalisé dans la phrase, il faut l'envisager sous la forme d'un terme PRO dans l'analyse des infinitives et d'un terme PRO arbitraire (sans rôle sémantique) lorsque le sujet « réel » est extraposé. Cette forme, PRO, joue le rôle syntaxique du sujet et en adopte les caractéristiques sémantiques.

29 En linguistique énonciative, certaines subordonnées ne donnent pas lieu à prédication lorsqu'elles expriment une caractéristique préconstruite et stable du référent : il y a alors une "co-prédication", avec une portée englobante. Le traitement des énoncés sans relation prédicative se fait au prix d'un élargissement de la définition de la prédication en tant que «structuration modalisée qui fonde l'énoncé ».

La linguistique cognitive quant à elle donne à comprendre l'absence de sujet par le mode de saisie de la scène ; en effet, le sujet cognitif a la possibilité d'appréhender une scène en en faisant un parcours sommatif ou bien un parcours séquentiel. Dans le parcours sommatif, le sujet cognitif aborde les moments de manière synthétique et globale, ce qui le libère de la nécessité d'isoler le sujet. Cela donne lieu notamment à des formes infinitivales et participiales, dans lesquelles l'absence de sujet n'est sans doute pas tant une ellipse qu'une indication donnée sur le mode de saisie de la scène.

\section{Mise en relation par les prépositions}

31 Certains enjeux de l'analyse des prépositions sont éclairés par la mise en regard des contributions: la délimitation de la catégorie des prépositions, le mode de mise en relation effectuée, l'existence de valeurs centrales, les principes conduisant des emplois « concrets » aux emplois abstraits.

Dans les ouvrages de grammaire portant sur l'anglais, les prépositions constituent une catégorie fermée, constituée de mots invariables introduisant des groupes nominaux et des formes nominales du verbe. Toutefois, en anglais, certains mots (comme after, before, since etc.) sont présents dans différentes catégories morphosyntaxiques et les difficultés de catégorisation surviennent rapidement au contact des textes : si les exemples cités figurent à la fois dans les catégories des prépositions, des conjonctions de subordination et des adverbes, de quelle catégorie relèvent-ils dans des énoncés comme I haven't seen them since. Doit-on considérer qu'il y a là un adverbe, ou bien une forme elliptique 
permettant de concevoir que since soit préposition (since that day) ou conjonction de subordination (since they have visited us). La prise en compte de la syntaxe de l'énoncé donne une première indication sur la nature du mot mais le débat est ouvert dès lors que la valeur prédicative de la préposition est prise en compte. Cela conduit à ne pas limiter la structure de la préposition à une binarité : X PREPOSITION Y, mais à envisager plus largement la valence de la préposition, comme on envisage la "valence» des verbes, terme forgé par Tesnière (1959: 238) qui envisageait le verbe comme une sorte d'atome "susceptible d'exercer son attraction sur un nombre plus ou moins élevé d'actants". Ainsi, les prépositions pourraient ne pas être limitées au schéma «bivalent» $\mathrm{X}$ PREPOSITION Y, mais être susceptibles d'entrer dans le schéma dit «monovalent » (X PREPOSITION), donnant lieu à une « définition large » (J.-M. Merle) de la catégorie. En structure « monovalente ", since est alors considéré comme préposition dans I haven't seen them since. De même, les propositions subordonnées complétives, dites aussi "nominales", dans la mesure où on peut les remplacer par un groupe nominal, entraînent la recatégorisation possible de la conjonction vers le statut prépositionnel: ainsi au début du corpus, le mot after peut être considéré comme préposition dans Ten days after the war ended, car la proposition est nominale : elle peut être remplacée par le groupe nominal that event. La contribution de J.-M. Merle, dans le paragraphe 4, montre aussi que la fonction du groupe prépositionnel peut être argumentale (et non pas seulement ciconstancielle) dans la mesure où la préposition introduit une facette du procès, faisant du groupe prépositionnel un co-prédicat.

Ces différentes analyses, permises par le cadre théorique de la TOPE, inscrivent singulièrement les prépositions dans le champ prédicatif, à plusieurs degrés, qui prennent en compte tant la préposition elle-même (et sa valence) que son rôle possible de co-prédicat.

Par ailleurs, la préposition peut créer une relation plus ou moins serrée avec le contexte droit : on peut évoquer les cas où la préposition introduit un complément du nom ayant un rôle déterminatif (" les fleurs du jardin sont magnifiques »; a hat with a veil l. 34) par opposition à une fonction circonstancielle et à une relation lâche («les fleurs, dans le jardin, attirent les papillons »; « Also I ought to warn Richard, at his office» 1.35).

Pour terminer les remarques d'ordre syntaxique, il est à noter que la position prototypique de la préposition, en amont de ce qu'elle introduit, n'est en rien une caractéristique définitoire ou contraignante: en anglais, la préposition peut être dissociée de ce qu'elle introduit et se trouver isolée en fin de proposition, comme dans les questions et les relatives. Elle est dite «orpheline» dans ce cas (par exemple of dans : what had she been thinking of?).

36 Au plan sémantique maintenant, différentes questions peuvent se poser. Le rapport entre le nombre de prépositions disponibles dans la langue et la variété de sens à exprimer a pour conséquence qu'une même préposition véhicule plusieurs sens. Les linguistes cherchent alors à comprendre pourquoi la même forme est requise : peut-on envisager qu'il y ait une valeur centrale pour chaque préposition, justifiant ainsi l'emploi du même mot ? Peut-on considérer par ailleurs qu'il y ait un emploi fondateur, à partir duquel des emplois dérivés se seraient développés? En quoi les connaissances en diachronie peuvent-elles étayer les réponses à ces deux questions, dans un dialogue fructueux entre diachronie et synchronie? Le rapprochement entre la contribution d'Anne Mathieu et celle de Jean-Rémi Lapaire est particulièrement intéressant puisque pour les trois prépositions plus particulièrement analysées en synchronie (by, of, for), Anne Mathieu 
indique pour chacune une racine indo-européenne relative à l'espace (respectivement envers un point central, une provenance, une location à l'avant) et un emploi initial d'ordre spatial pour ces prépositions. Les emplois temporels se développent ultérieurement. En linguistique cognitive, l'information perceptuelle et sensorielle fournie par le corps dans son espace est à la source des représentations cognitives, et donc linguistiques. La perception de l'espace donne lieu aux repérages statiques, dynamiques et directionnels. La conception de l'espace semble préalable à celle de la temporalité (Markman, 1989). Une réelle cohérence apparaît entre diachronie, cognition et acquisition, qui semble favoriser l'hypothèse selon laquelle le sens fondateur des prépositions soit le sens spatial.

Concernant l'extension vers des emplois non spatiaux et abstraits, les hypothèses peuvent encore s'appuyer sur la diachronie, avec l'apparition secondaire de sens abstraits et une grammaticalisation probable au bout du processus de diversification des emplois; ce pourrait être le cas de of selon Anne Mathieu; dont Huddleston \& Pullum (2002: 658) considèrent également que of est la plus hautement grammaticalisée de toutes les prépositions (« the most highly grammaticised of all prepositions »), ne conservant que le sens de relateur. Au cœur du processus de diversification, la linguistique cognitive fait l'hypothèse de la métonymie : la scène perçue donne lieu à une schématisation et n'en retient que quelques traits distinctifs. Cette schématisation est adossée à la nécessaire simplification de la réalité pour éviter une surcharge cognitive, ainsi qu'au principe de saillance, qui conduit l'énonciateur à sélectionner certains traits selon des critères culturels et interlocutifs. Une autre opération, la projection métaphorique, permettrait de rendre compte, toujours en linguistique cognitive, de l'extension des emplois spatiaux aux emplois abstraits (in shock 1.38) par construction d'un espace abstrait (Radden \& Dirven, cités par J.-R. Lapaire) ; à cette hypothèse, Jean-Rémi Lapaire préfère l'hypothèse d'un "espace épistémique", propre à l'énonciateur, puisque ce sont les conceptualisations incarnées chez l'énonciateur qui déterminent l'emploi de la préposition. Cette approche est convaincante par la cohérence qu'elle présente avec la corporéité de l'individu, ses perceptions sensorielles et les aspects gestuels du discours individuel: ces derniers procurent un accès privilégié aux conceptions et aux connaissances exprimées par l'énonciateur.

$\mathrm{Au}$ plan sémantique enfin, le fait de mettre en relation des termes implique l'établissement d'un repérage entre eux. Cette prise en compte constitue un apport original de la TOPE. Dans l'emploi des prépositions, J.-M. Merle relève trois types de repérage : lorsque le groupe prépositionnel est co-prédicatif, la préposition instaure une relation d'incidence à $\mathrm{X}$ (premier repérage), et se trouve donc indexée sur la situation d'énonciation (second repérage). La relation peut également ordonner $\mathrm{X}$ par rapport à $\mathrm{Y}$, dans un rapport possible de cause, de temps, d'origine, d'effet etc. Il est à noter de plus, que parmi les quatre valeurs possibles de l'opérateur de repérage proposé dans la TOPE (l'opérateur $\underline{\varepsilon}$ ), seules deux sont peuvent être exploitées dans les relations prépositionnelles: l'identification et la différenciation. En effet, les deux autres sont incompatibles avec le statut de relateur de la préposition: la valeur de rupture et la valeur *, qui combine les trois autres, dont la rupture, de ce fait. Les repérages par identification ne sont pas représentés dans le texte mais peuvent être illustrés par work to death (work = die), be chased to exhaustion (be chased = be exhausted), alors que la valeur de différenciation est bien représentée dans le corpus, avec par exemple : a hat with a veil (tout en étant différents, veil et hat sont reliés). 

jour.

\section{Prédication et prépositions : points communs et différences ?} particulièrement visible dans la langue cette activité linguistique fondamentale, voire fondatrice, qu'est la mise en relation. La catégorie des prépositions n'est pas moins « accessoire » que la prédication, pour faire écho à un volume consacré à la préposition (Morel, 1997). Les deux formes étudiées semblent avoir bien plus de points communs que de différences de ce point de vue.

41 La différence la plus notable concerne le fait que l'analyse des prépositions par la linguistique cognitive s'appuie sur les fondements (perception / conception) de cette théorie, alors que la grammaire de constructions, qui en est un prolongement particulièrement utile pour les hypothèses sur l'acquisition du langage, permet de rendre compte de la prédication. Les prépositions quant à elles entretiennent un lien fort avec la spatialité. Or, comme le souligne J.-R. Lapaire, la linguistique cognitive était initialement dénommée space grammar et les prépositions ont constitué un axe d'investigation privilégié. Il est donc logique que l'étude des prépositions proposée ici soit ancrée dans l'approche perceptuelle et conceptuelle de la linguistique cognitive.

42 Pour ce qui est des points communs, nous relevons pour les deux thématiques un rôle prédicatif, la participation à un processus de grammaticalisation, une possible incomplétude.

43 Par leur rapprochement sous la bannière d'un rôle prédicatif, des outils d'analyse communstels que la valence et la relation d'incidence peuvent être convoqués. D'inspiration structuraliste pour la valence et d'inspiration guillaumienne pour l'incidence, ces deux concepts permettent de développer les aspects syntaxiques et sémantiques des formes à l'étude. Le rôle prédicatif peut se complexifier avec le statut coprédicatif, auquel les deux études menées dans le cadre de la TOPE font référence.

Prédication et prépositions sont soumises à un processus de grammaticalisation. L'auxiliaire DO est le signe de la prédication mais lorsque celle-ci va de soi, DO est omis (réduit phonologiquement à Ø), ce qui est le cas des énoncés affirmatifs complets. De même, le sens de la préposition of est limité à celui de mise en relation et donc à sa fonction grammaticale: l'atténuation sémantique va de pair avec l'affaiblissement vocalique voire avec l'effacement phonologique lorsque la mise en relation est repoussée à l'arrière-plan (préposition Ø dans Sunday papers).

45

Quant au fait que la prédication et les prépositions soient des lieux d'incomplétude par la nature même de leur rôle dans le langage et dans la langue, thématique que nous avons évoquée en introduction, cela n'est pas pris en compte explicitement par les études présentées mais transparaît dans les diverses propositions faites pour expliquer les cas dans lesquels un élément semble manquer; les différentes études montrent que cet élément, qui peut être le sujet dans la prédication ou le terme introduit par la préposition, est récupérable, soit par l'insertion d'une forme PRO dans la structure, soit par co-prédication, soit par ellipse. Cette démarche atteste implicitement de la complétude obtenue par la mise en relation. 
Que soient remerciés Evelyne Chabert (Université Grenoble Alpes), Caroline David (Université Paul Valéry, Montpellier) et Jérôme Puckica (Université Grenoble Alpes) pour leur participation au comité scientifique de ce volume.

\section{BIBLIOGRAPHIE}

Amiot D. (2006). « 2. Préposition et préfixes ». Modèles linguistiques [En ligne], 53 | 2006, mis en ligne le 01 février 2015, consulté le 01 octobre 2016. URL : http://ml.revues.org/515 ; DOI : $10.4000 / \mathrm{ml} .515$

Col G. (2008) « 'Relation' ou 'intégration' prédicative ? La prédication comme principe d'émergence du sens », Faits de Langues, n³1/32, J.-M. Merle (dir.), p. 33-43.

Franckel J.-J \& Paillard D. (2007). Grammaire des prépositions. Tome 1. Paris : Ophrys.

Markman E.M. (1989). Categorization and Naming in Children. Cambridge, Mass. : MIT Press.

Huddleston R. et Pullum G.K. (2002). The Cambridge Grammar of the English Language. Cambridge : Cambridge University Press.

Merle J.-M. (dir., 2008). La prédication. Faits de langues, n³1-312, Paris : Ophrys.

Morel M.-A. (dir., 1997). La préposition : une catégorie accessoire ? Faits de langues, $\mathrm{n}^{\circ}$ 9, Paris : Ophrys.

Platon, Le Sophiste, traduction N. Cordero, Paris : Garnier Flammarion, 1993, p. 191-197.

Riegel M., Pellat Jean-Christophe \& Rioul René (1998). Grammaire méthodique du français, Paris : Presses Universitaires de France.

Tesnière L. (1959). Eléments de syntaxe structurale. Paris : Klincksieck. 